Protocols

\title{
Endocytosis and intracellular traffic of cholesterol-PDMAEMA liposome complexes in human epithelial-like cells
}

\author{
F. Szymanowski ${ }^{a}$, A.A. Hugo ${ }^{a}$, P. Alves ${ }^{c}$, P.N. Simões ${ }^{c}$, A. Gómez-Zavaglia ${ }^{a}$, \\ Pablo F. Pérez ${ }^{\mathrm{a}, \mathrm{b}, *}$ \\ a Centro de Investigación y Desarrollo en Criotecnología de Alimentos (CIDCA,-CCT-CONICET La Plata), 47 y 116 (s/n) B1900AJI. La Plata, Argentina \\ b Cátedra de Microbiología, Facultad de Ciencias Exactas (FCE), UNLP, 47 y 115 (s/n) 1900 La Plata, Argentina \\ c Department of Chemical Engineering, University of Coimbra, P-3030-790 Coimbra, Portugal
}

\section{A R T I C L E I N F O}

\section{Article history:}

Received 30 January 2017

Received in revised form 4 April 2017

Accepted 29 April 2017

Available online 1 May 2017

\section{Keywords:}

\section{CHO-PDMAEMA}

Polymer-lipid complexes

Liposome internalization

Endocytic pathways

Caveolae

\begin{abstract}
A B S T R A C T
Liposomes are generally used as delivery systems, as they are capable of encapsulating a wide variety of molecules (i.e. plasmids, recombinant proteins, therapeutic drugs). However, liposomal drug delivery have to fulfill different requirements, such as the effective internalization by the target cells and avoidance of the degradative activity of the intracellular compartments. The use of polymer lipid complexes (PLCs), by including different polymers in the liposome formulation, could improve internalization and intracellular release of drugs. The aim of the present work is to study the mechanisms of cellular uptaking and the intracellular trafficking of PLCs formed with cholesterol-poly(2-(dimethylamino)ethyl methacrylate) CHO-PDMAEMA and lecithin (LC CHO-PD). Calcein-loaded liposomes were used to determine cellular uptake and intracellular localization by flow cytometry and confocal microscopy. Incorporation of CHOPDMAEMA to lecithin liposomes enhanced the internalization capacity of PLCs. Internalization of PLCS by human epithelial-like cells (HEK-293) diminished at $4{ }^{\circ} \mathrm{C}$, suggesting uptake by endocytosis. PLCs showed no co-localization with acidic compartments after internalization. Experiments with endocytosis inhibitors and co-localization of liposomes and albumin, suggested the caveolae endocytic pathway as the most probable route for intracellular trafficking of PLCs.

In this work, we demonstrated an efficient uptake of LC CHO-PDs by human epithelial-like cells (HEK293) through the non-degradative caveolae endocytic pathway. The mode of internalization and the intracellular fate of liposomes under study, suggest a promising use of LC CHO-PDs as drug delivery systems.
\end{abstract}

(c) 2017 Elsevier B.V. All rights reserved.

\section{Introduction}

Liposomes are self-assembledlipidic bilayered vesicles able to encapsulate hydrophobic or hydrophilic molecules [1]. These nanosized particles play an important role as drug delivery systems, as they can encapsulate a wide variety of low molecular weight drugs, as well as biopharmaceutical agents, including recombinant pro-

Abbreviations: CHO-PDMAEMA, cholesterol-poly(2-(dimethylamino)ethyl methacrylate); DMEM, Dulbecco's Modified Eagle Medium; FACS, fluorescence activated cell sorting; HEK-293 cells, human embryonic kidney cells; LC CHO-PD liposomes, liposomes formed with lecithin and CHO-PDMAEMA; PBS, phosphate buffered saline; PLCs, polymer lipid complexes.

* Corresponding author at: Centro de Investigación y Desarrollo en Criotecnología de Alimentos (CIDCA,-CCT-CONICET La Plata), 47 y 116 (s/n) B1900AJI. La Plata, Argentina.

E-mail address: pfp@biol.unlp.edu.ar (P.F. Pérez). teins, antisense oligonucleotides, and plasmids [2]. Liposomes and other lipid-based nanoparticles (LNPs), which mimic the structure of lipoproteins, are specially suitable to deliver hydrophobic drugs or probes to a disease site in vivo [3]. Positive properties of biocompatibility, low immunogenicity, low toxicity, low cost of fabrication and ease of functionalization have driven liposomes to the forefront of nanoparticle delivery systems [4]. However, conventional liposome formulations generally suffer from poor cell penetration and low stability in bloodstream; therefore, they need to be modified to improve their performance in vivo $[5,6]$.

Different endocytic pathways could be involved in the incorporation of liposomes into eukaryotic cells $[7,8]$, and the direct fusion of liposomes with plasma membrane can occur as well [9]. Endocytosis process can be classified in two broad categories: phagocytosis (the uptake of large particles and microorganisms) and pinocytosis (the uptake of fluid and solutes) [10]. Phagocytosis is typically restricted to specialized mammalian cells, whereas pinocytosis 
occurs in all cells. At least four morphologically distinct pinocytic pathways have been characterized: clathrin-mediated endocytosis, caveolae endocytosis, macropinocytosis, and clathrin/caveolaeindependent endocytosis [11].

Clathrin-mediated endocytosis involves a strong binding of ligands to specific cell surface receptors as a first step. This results in the clustering of ligand-receptor complexes in coated pits on plasma membrane. These complexes, are formed by the assembly of cytosolic coat proteins, being, clathrin the most important one $[10,12]$. The coated pits then invaginate and pinch off from the plasma membrane to form intracellular clathrin-coated vesicles. Next, clathrin coats depolymerize, resulting in early endosomes that in turn mature in late endosomes and finally fuse with lysosomal compartments. This pathway involves acidification of intracellular compartments that decreases to $\mathrm{pH}$ 5.9-6.0 in late endosomes and drops to $\mathrm{pH} 5$ in lysosomes [13]. Epidermal growth factor receptor, the transferrin receptor and transferrin, as well as LDL lipoprotein are ligands typically incorporated by clathrinmediated endocytosis pathway.

Caveolae endocytosis are mediated by caveolae (small, hydrophobic membrane microdomains rich in cholesterol and glycosphingolipids, associated with a family of cholesterol-binding proteins called caveolins) [14,15]. Caveolae are involved in endocytosis of ligands such as simian virus 40 (SV40), cholera toxin B and albumin [16]. As caveolar uptake is a nonacidic and nondigestive route of internalization, caveosomes are directly transported to the Golgi and/or endoplasmic reticulum, thus avoiding fusion with lysosome and further degradation [17].

Macropinocytosis refers to the formation of large endocytic vesicles, called macropinosomes, generated by actin-driven invagination of the plasma membrane [18]. Macropinosomes have no coat and do not concentrate receptors. Because they are relatively large, macropinocytosis is an efficient route for the nonselective endocytosis of soluble macromolecules. In macrophages, macropinosomas become acidified, and then completely merge into the lysosomal compartment [19]. In non-phagocytic cells, they constitute a distinct vesicle population, which eventually recycles most of its contents back to the cell surface without fusing with lysosomes [18].

Clathrin/caveolae-independent endocytosis includes endocytic pathways dependent on lipid rafts for vesicle formation such as flotillin-mediated endocytosis and the GEEC (glycosylphosphatidylinositol -enriched endosomal compartments) pathway [20]. Molecules internalized by this pathway do not route to acidic compartments [4].

Since the fate of internalized molecules is closely related to the uptake mechanism, targeting these mechanisms could improve the performance of liposomal delivery. In this sense, the incorporation of liposomes through non-clathrin endocytic pathways constitutes a desirable strategy.

In a previous work, we demonstrated that incorporation of cholesterol-poly(2-(dimethylamino)ethyl methacrylate) (CHOPDMAEMA) into lecithin liposomes, increased their stability at neutral $\mathrm{pH}$, the uptake by eukaryotic cells, the release of their content in mild acidic conditions [21]. These promising results encouraged us to go deeper in the characterization of PLCs using in vitro cell models. The present work reports the mechanisms of cellular uptake and intracellular trafficking of PLCs.

\section{Materials and methods}

\subsection{Preparation of liposomes}

Cholesterol-poly(2-(dimethylamino)ethyl methacrylate)(CHOPDMAEMA) was synthesized by atom transfer radical polymer- ization according to Alves et al. [21]. Bare lecithin liposomes (LC) and PLCs were prepared using the hydration film method [22]. Briefly, lecithin (6.25 mM) (Calbiochem, Merck KGaA, Darmstadt, Germany) and CHO-PDMAEMA were dissolved in chloroform $(0.4 \mathrm{mM})$. PLCs were prepared by mixing lecithin and $\mathrm{CHO}-$ PDMAEMA in a lipid molar ratio of 0.10 (LC CHO-PD/PLCs) and further dried under a nitrogen stream. The dried lipid/polymer film was then rehydrated with a buffered solution of $60 \mathrm{mM}$ calcein (Sigma-Aldrich Co., St. Louis, MO) in $10 \mathrm{mM} \mathrm{HEPES} \mathrm{(pH} \mathrm{7.0),} \mathrm{and}$ vigorously stirred above the transition temperature $\left(\mathrm{ca} .37^{\circ} \mathrm{C}\right)$, to allow the liposome formation. The resulting multillamelar vesicles were collected by centrifugation at $14,000 \mathrm{~g}(12,300 \mathrm{rpm})$ for $10 \mathrm{~min}$ and then washed twice with $100 \mathrm{mM}$ HEPES ( $\mathrm{pH}$ 7.0) to remove non-entrapped calcein. Calcein fluorescense is self-quenched at these concentration. Calcein loaded multillamelar liposomes were used in all the subsequent experiments.

\subsection{Cell culture}

The human epithelial-like cells (HEK-293) were cultured in Dulbecco's Modified Eagle Medium (DMEM, GIBCO BRL Life Technologies, Rockville, MD, USA) supplemented with: 10\% (v/v), heat-inactivated $\left(30 \mathrm{~min} / 60^{\circ} \mathrm{C}\right)$, foetal bovine serum (Internegocios S.A, Mercedes, Bs. As. Argentina), 1\% (v/v) non-essential amino acids (GIBCO BRL Life Technologies, Rockville, MD, USA) and $1 \%(\mathrm{v} / \mathrm{v})$ penicillin-streptomycin solution $[100 \mathrm{U} / \mathrm{mL}$ penicillin G,100 $\mu \mathrm{g} / \mathrm{mL}$ streptomycin, (GIBCO BRL Life Technologies, Rockville, MD, USA)]. Cells were incubated at $37^{\circ} \mathrm{C}$ in $5 \% \mathrm{CO}_{2}$ and 95\% air atmosphere.

\subsection{Uptake of liposomes by eukaryotic cells}

HEK-293 cells were seeded in 24-well culture plates (density ca. $2 \times 10^{5}$ cells $/ \mathrm{mL}$ ) and incubated to early post confluence at $37^{\circ} \mathrm{C}$, as indicated in the previous section.

Monolayers were washed with phosphate saline buffer [PBS $\left(\mathrm{K}_{2} \mathrm{HPO}_{4} 0.144 \mathrm{~g} / \mathrm{L} ; \mathrm{NaCl} 9.00 \mathrm{~g} / \mathrm{L} ; \mathrm{Na}_{2} \mathrm{HPO}_{4} 0.795 \mathrm{~g} / \mathrm{L}\right), \mathrm{pH}$ 7], and fresh medium containing calcein-loaded liposomes was added to each well. Concentration of liposomes was assessed by counting appropriate dilutions in a haemocytometer and adjusted to obtain a ratio liposome/cell of approximately 100:1. After being incubated for 30 min, cells were washed twice with PBS to remove free noninternalized liposomes. For assays carried out in the presence of endocytosis inhibitors, each inhibitor was incubated for $1 \mathrm{~h}$ with cells prior to the addition of calcein-loaded liposomes.

Following the incubation with liposomes, cells were washed and removed by trypsination. Afterward they were transferred to FACS tubes containing PBS and acquired by flow cytometry. The endocytosis inhibitors used were: wortmannin $(25 \mathrm{nM})$ and cytochalasin $\mathrm{D}(9.8 \mu \mathrm{M})$, which inhibit the macropinocytosis process; genistein $(200 \mu \mathrm{M})$, nystatin and $\beta$ cyclodextrin $(4.4 \mathrm{mM})$, which inhibit caveolae endocytosis, and finally $\mathrm{NH}_{4} \mathrm{Cl}(30 \mathrm{mM})$ and chloropromazine $(10 \mu \mathrm{M})$ which inhibit clathrin pathways. Wortmannin, cytochalasin $D$, genistein, $\beta$ cyclodextrin were purchased from Sigma-Aldrich Co., (St. Louis, MO. USA); chlorpromazine was purchased from Sanofi-Aventis (Gentilly, France) and nystatin was purchased from Denver Pharma Laboratories (Buenos Aires, Argentina). Cell viability in presence of endocytosis inhibitors was determined by adding propidium iodine (PI) (final concentration $20 \mu \mathrm{M}$ ) (Thermo Fisher, Waltham MA, USA) prior to flow cytometer acquisition.

Flow cytometry analysis was performed in a FACSCalibur flow cytometer by using Cell Quest software (Becton Dickinson, Mountain View, CA, USA). Green (FL1) and red (FL2) fluorescence of 10,000 events were analyzed for each sample. 


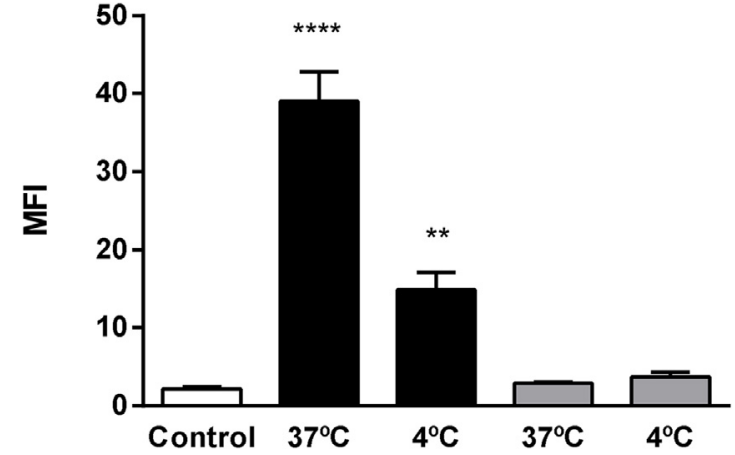

Fig. 1. Internalization of bare liposomes and calcein PLCs of lecithin and CHOPDMAEMA (LC CHO- PD) by HEK-293 cells. Results are expressed as the mean of fluorescence intensity (MFI). Black bars represent the MFI of cells incubated with LC CHO-PDMAEMA liposomes, grey bars represent the MFI of cells incubated with bare liposomes, white bars represent the MFI of cells control cells (without liposomes). Cells were incubated during $30 \mathrm{~min}$ with liposomes at $4{ }^{\circ} \mathrm{C}$ or $37^{\circ} \mathrm{C}$. Values were obtained by flow cytometry. Asterisks represent significant differences: ${ }^{*} \mathrm{p}<0.1$; ${ }^{* *}$ $\mathrm{p}<0.05 ;^{* * *} \mathrm{p}<0.01 ;{ }^{* * * *} \mathrm{p}<0.001$ as compared to control.

\subsection{Intracellular localization of liposomes}

Cells cultured on glass coverslips (Assistent, Glaswarenfabrik $\mathrm{KG}$, Sondheim, Germany) were incubated for $30 \mathrm{~min}$ with DMEM containing calcein-loaded liposomes and the endocytic marker under study [i.e. transferrin Alexa 596 or albumin Alexa 647 (Invitrogen, Eugene OR, USA) at concentrations of 10 and $30 \mu \mathrm{g} / \mathrm{mL}$, respectively]. After incubation, coverslips were washed twice with PBS, fixed with $3 \% \mathrm{w} / \mathrm{v}$ paraformaldehyde and mounted with antifading mounting media (Dako- Agilent Technologies, Carpinteria, C.A. USA).

To assess localization of liposomes in acidic intracellular compartments, monolayers were incubated for 30 min with DMEM containing calcein-loaded liposomes, washed with PBS as described above, and then incubated for $30 \mathrm{~min}$ with Lysotracker DND-99 $10 \mu \mathrm{M}$ (Molecular Probes, Oregon, USA).

Confocal images were taken by using a Leica laser scanning microscope (LSM) (LEICA TCS SP5, Leica Microscopy Systems Ltd. $\mathrm{CH}$ Heerbrugg, Switzerland), and image analysis was done using the Leica Application Suite X (Leica Microsystems) and Fiji - ImageJ (NIH, USA) software.

\subsection{Statistics}

All assays were performed in triplicate and in three independent assays. Means were compared by the Tukey's test $(P<0.05)$. Analysis of variance (ANOVA) was carried out by using InfoStat 2008 (InfostatGroup/FCA. National University of Cordoba. Ed. Brujas, Cordoba, Argentina).

\section{Results}

\subsection{Cellular uptake}

At $37^{\circ} \mathrm{C}$, HEK-293 cells efficiently incorporated PLCs. Interestingly; values of mean fluorescence intensity were significantly lower at $4{ }^{\circ} \mathrm{C}$ (Fig. 1 ).

To elucidate the endocytic pathways related with the internalization of PLCs in cells, different internalization inhibitors were employed (Fig. 2). The internalization of LC CHO-PD liposomes showed a significant decrease as compared with controls $(\mathrm{p}<0.05)$ in the presence of caveolae inhibitors genestein, nystatin and $\beta$ cyclodextrin (Fig. 2A). In contrast, internalization was not affected neither by clathrin $\left(\mathrm{NH}_{4} \mathrm{Cl}\right.$, chlorpromazine) nor by macropinocyto- sis inhibitors (wortmannin and cytochalasin D) (Fig. 2A). Viability of HEK-293 cells was not affected by the inhibitors in the conditions of the present study as no significant increase in the ratio of PI positive cells as compared to controls, was observed (Fig. 2B).

\subsection{Intracellular localization}

Fluorescent microscopy analysis showed a good correlation with flow cytometry results, indicating that LC CHO-PD liposomes interacted better with HEK-293 cells as compared with bare liposomes (Fig. 3).

To confirm the role of caveolae in the incorporation of LC CHO-PD10 liposomes by the cells, we used calcein-loaded PLCs in the uptake assay along with lysotracker (a fluorescent marker of intracelullar acid compartments) and different fluorescent proteins such as transferrin and albumin that traffic to different compartments (Fig. 4). As mentioned in the introduction, transferrin is incorporated by cells using clathrin mediated endocytosis, whereas albumin is typically incorporated by the caveolae pathway.

Incubation of HEK-293 cells with lysotracker or transferrin showed no co-localization of liposomes and these probes after $30 \mathrm{~min}$. On the other hand, there was co-localization of liposomes and albumin, indicating a similar internalization pathway and intracellular traffic (Fig. 4).

\section{Discussion}

To determine the effectiveness of liposomes as drug-delivery systems, intracellular trafficking must be carefully assessed. In our previous work, we designed stimuli-responsive multillamelar PLCs by the incorporation of CHO-PDMAEMA in lecithin liposomes. The polymer incorporation had a stabilizing effect on the PLCs in buffer, and a pH-dependent release of liposome content [21]. In the present work, we aimed to gain insight into the internalization and cellular traffic of multillamelar PLCs in epithelial like cells. We selected the use of multilamellar liposomes based on their high efficiency of encapsulation and a relative slow release of the entrapped compounds [23,24]. On the contrary, small unilamellar liposomes usually have a very weak capacity to trap drugs and usually exhibited a burst release of its content $[23,24]$.

PLCs had a noticeable better internalization in eukaryotic cells than bare liposomes (Fig. 1). In previous studies, we determined that the incorporation of CHO-PDMAEMA to bare liposomes induces a positively charged surface, which may promote the association of liposomes with negatively charged eukaryotic cell surfaces [21]. In general, PDMAEMA shows a better rate of uptake and less toxicity than other cationic polymers such as polyethylenimine [25].

In the present study, cellular uptake of multilamellar PLCs noticeably decreased when the incubation temperature decreased from $37^{\circ}$ to $4{ }^{\circ} \mathrm{C}$. It is known that endocytosis is an energy dependent mechanism, and, therefore, it can be strongly inhibited by lowering the temperature or by using ATP-depleting metabolic inhibitors [26]. Our results are in agreement with previous works, which have established that the majority of cells internalize liposomes through an endocytic pathway [27]. Even very small unilamellar liposomes can be taken up by cells using an energydependent pathway [28]. Moreover, it has been shown that the polymer PDMAEMA is actively taken up by cells through endocytosis [29].

PLCs incorporation by eukaryotic cells was significantly decreased in the presence of inhibitors of caveolae-mediated endocytosis (Fig. 2). This pathway involves the formation of caveolae, which are small membrane microdomains rich in cholesterol and glycosphingolipids that are also associated with caveolins 

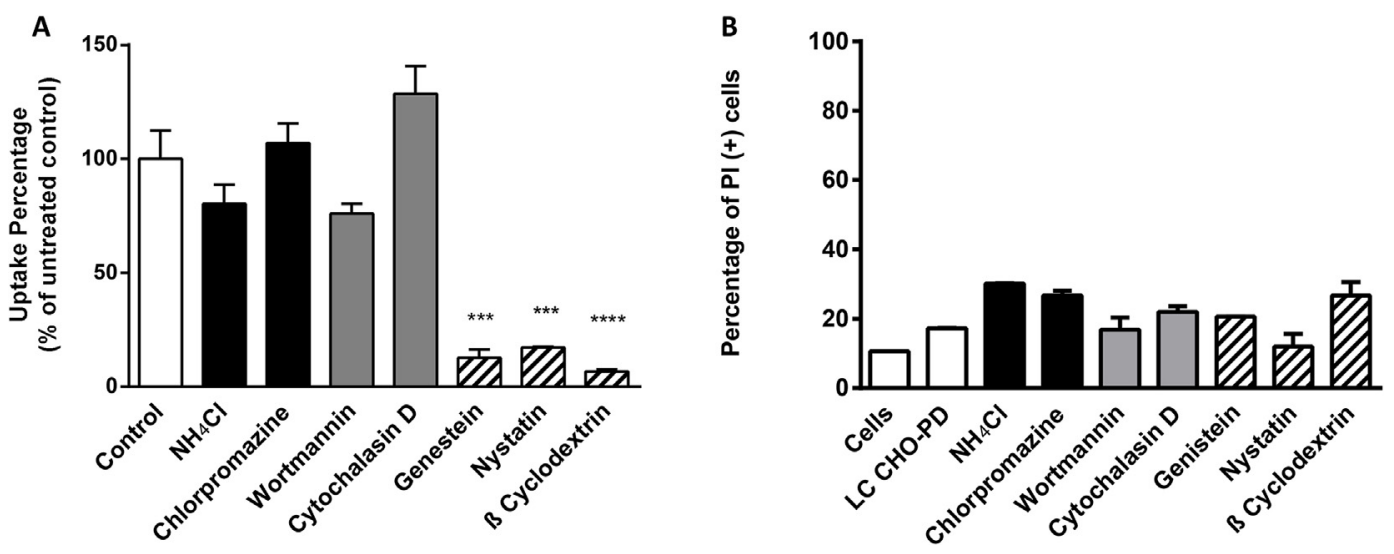

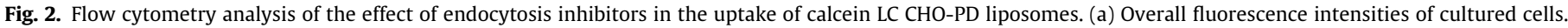

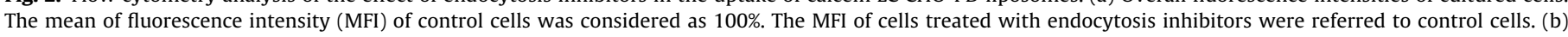

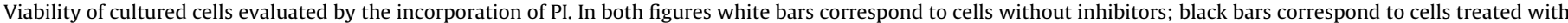

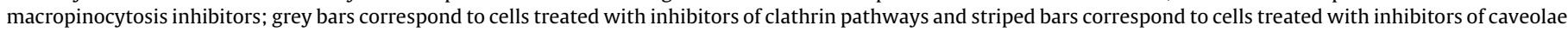
pathways. Asterisks represent significant differences: ${ }^{*} \mathrm{p}<0.1$; ${ }^{* *} \mathrm{p}<0.05$; and ${ }^{* * *} \mathrm{p}<0.01$ as compared to control.
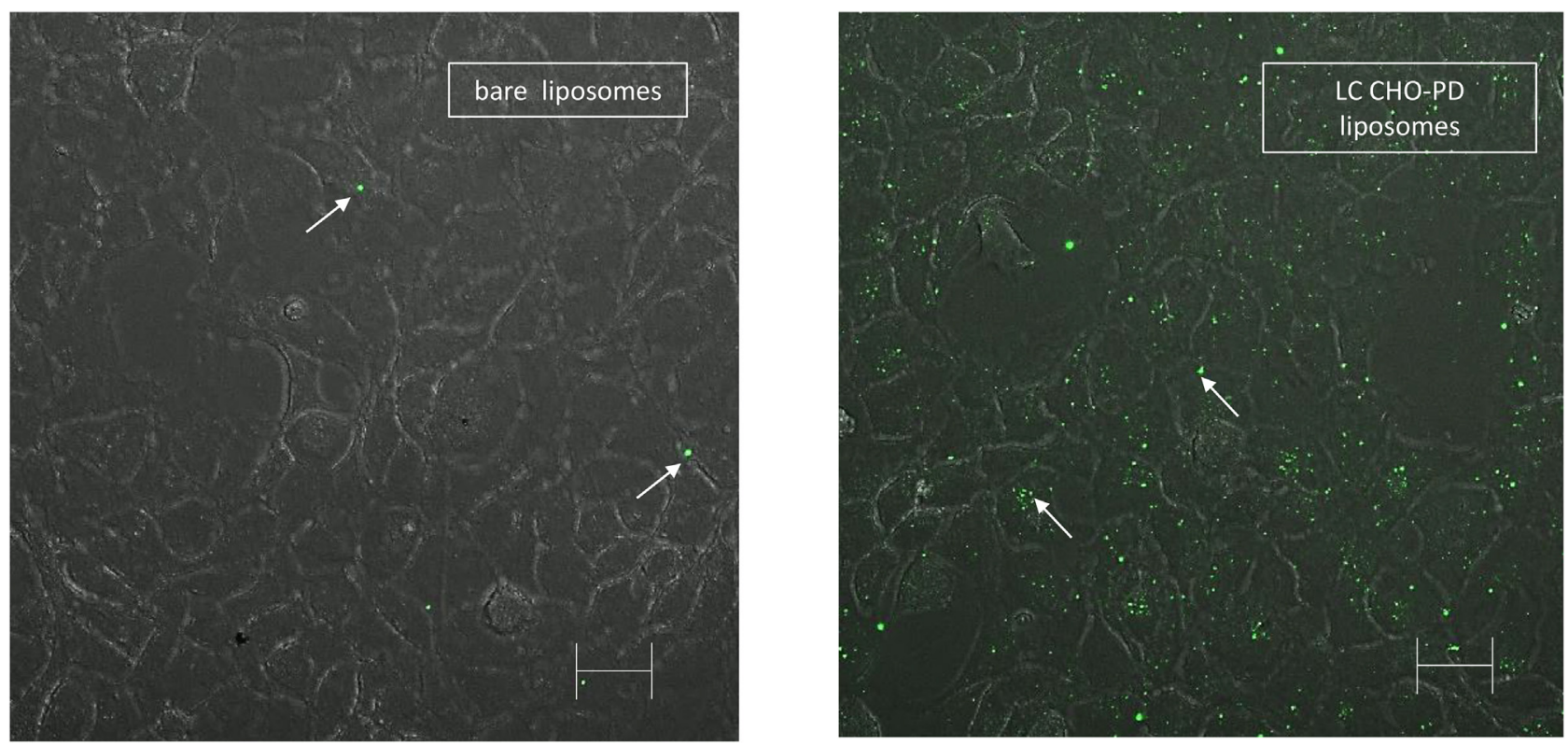

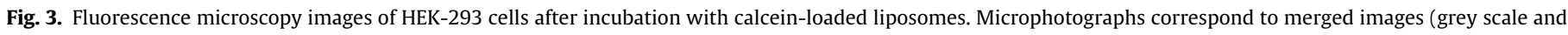

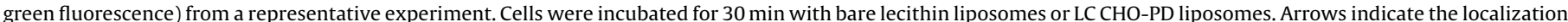

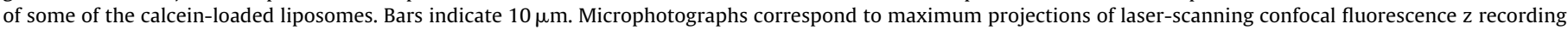
$(0.3 \mu \mathrm{m}$ step size). Pinhole 1 A.U. (For interpretation of the references to color in this figure legend, the reader is referred to the web version of this article.)

[15]. Drugs that specifically binds to cholesterol, such as metil $\beta$ cyclodextrin and nystatin, perturb the internalization related to caveolae [11]. Other non-related drugs, such as genistein (a tyrosine kinase inhibitor), can also inhibit caveolae-mediated internalization [30]. Molecules incorporated through this pathway are initially associated with the cell membrane and then become trapped in relative stationary caveolae, which deliver their content to the endoplasmic reticulum or the Golgi without acidification [17]. Analysis by confocal microscopy correlates with the results obtained with the endocytosis inhibitors (Fig. 4). Confocal images showed no co-localization of PLCs neither with lysotracker (marker of lysosomal compartments) nor transferrin (marker of clathrinmediated endocytosis) (Fig. 4). These results suggest that there is no incorporation of liposomes by the clathrin pathway, which involves routing to acidic lysosomal compartments. It is important to point out that we found an evident colocalization of PLCs and albumin thus suggesting that PLCs are internalized by a caveolae- dependent endocytic pathway (Fig. 4). It has always been accepted that the size of nanoparticles may be an important factor affecting the endocytic pathway employed in the cellular uptake [31]. Thermodynamic studies have established an optimal radius of $25 \mathrm{~nm}$ for internalization of nanoparticles [31]. The large particles are most likely to be engulfed via macropinocytosis. The size of vesicles involved in clathrin mediated endocytosis is about $100 \mathrm{~nm}$, while the size of those involved in caveolae mediated endocytosis is about $60-80 \mathrm{~nm}$ [32]. In our previous work, we determined the size of the PLCs, which were about $80 \mathrm{~nm}$ [21]. The diameter of the PLCs under study are in agreement with the described sized for caveolae internalization and with our current results. On the other hand, some researchers suggest that the size may not be such important compared to other factors in the pathway selection of nanoparticles entry into cells [33]. In this work we investigated the cellular uptake of multilamellar PLCs The obtaining of smaller unilamel- 

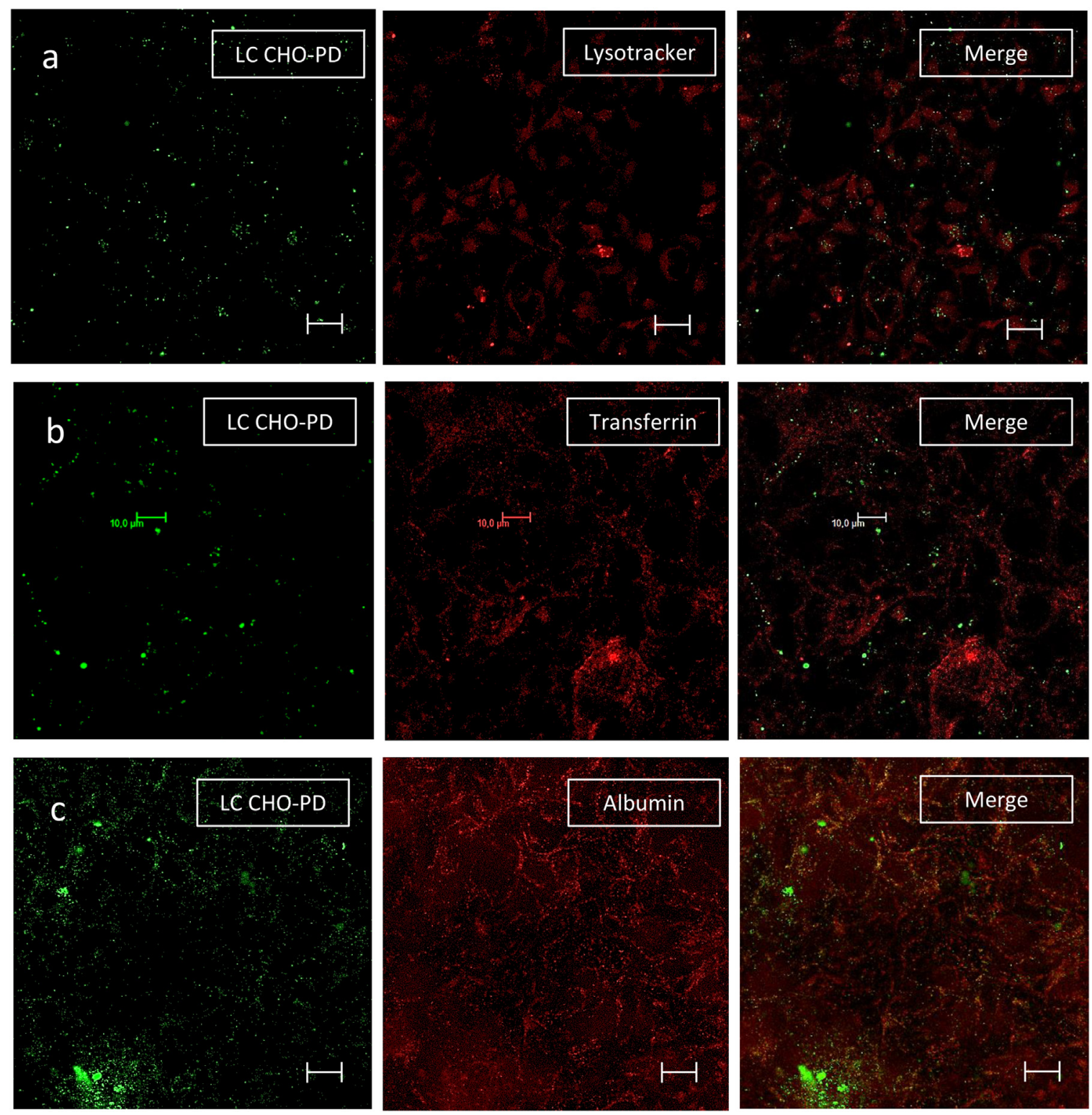

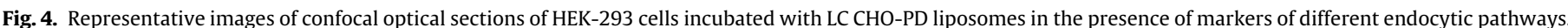

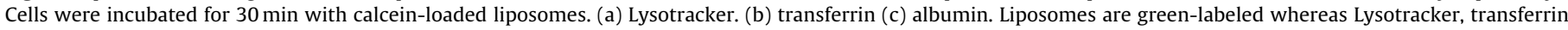

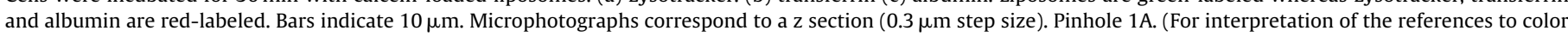
in this figure legend, the reader is referred to the web version of this article.)

lar liposomes with similar composition could probably modify the endocytic pathway employed.

It is known that cell-uptake mechanisms are closely linked with the intracellular trafficking. In this context, routing of liposomes to non-degradative intracellular compartments could be relevant to improve drug delivery [16]. In this work, we demonstrated for the first time, a high efficiency of incorporation of PLCs by epithelial like cells by a caveolae-dependent endocytic pathway. These findings could be related to better stability of cargo molecules intended for therapeutic applications.

\section{Conclusions}

This work reports promising results regarding the interaction with eukaryotic cells of PLCs formulated with CHO-PDMAEMA and lecithin. The incorporation of CHO-PDMAEMA enhanced the internalization of the PLCs. Moreover, PLCs traffic through the non-degradative caveolae endocytic pathway, thus preserving lipo- some cargo from an acidic environment. Our findings showed that lecithin CHO-PDMAEMA liposomes constitute an interesting alternative for drug delivery of bioactive molecules sensitive to low $\mathrm{pH}$ that must be protected from acid degradation.

\section{Acknowledgments}

A.A.H. A.G.Z and P.F.P. are researchers of the Consejo Nacional de Investigaciones Científicas y Técnicas CONICET (Argentina); F.S. is a fellow from CONICET; P.A. is a postdoctoral fellow in CIEPQPF and would like to thank FCT (Grant no: SFRH/BPD/69410/2010) for her grant. P.F.P. is Professor of Microbiology at the Department of Biological Sciences of the Facultad de Ciencias Exactas (UNLP, Argentina). The present work was partially financed by the Agencia Nacional de Promoción Científica y Tecnológica (ANPCyT) (Projects PICT 2013-1205, PICT 2014-0912), CONICET (Project PIP 
2013-0577) and Universidad Nacional de La Plata (Project 11/X670) (Argentina).

\section{References}

[1] A. Jone, Liposomes: a short review, J. Pharm. Sci. Res. 5 (2013) 181-183.

[2] A. Akbarzadeh, R. Rezaei-sadabady, S. Davaran, S.W. Joo, N. Zarghami, Liposome: classification, preparation, and applications, Nanoscale Res. Lett. 8 (2013) 1, http://dx.doi.org/10.1186/1556-276X-8-102.

[3] N. Anikeeva, Y. Sykulev, E.J. Delikatny, A.V. Popov, Core-based lipid nanoparticles as a nanoplatform for delivery of near-infrared fluorescent imaging agents, Am. J. Nucl. Med. Mol. Imaging 4 (2014) 507-524.

[4] K. Cleal, L. He, P.D. Watson, A.T. Jones, Endocytosis, intracellular traffic and fate of cell penetrating peptide based conjugates and nanoparticles, Curr. Pharm. Des. 287 (2013) 2878-2894.

[5] N. Oku, Anticancer therapy using glucuronate modified long-circulating liposomes, Adv. Drug Deliv. Rev. 40 (1999) 63-73.

[6] Y. Tseng, J. Liu, R. Hong, Translocation of liposomes into cancer cells by cellpenetrating peptides penetratin and tat: a kinetic and efficacy study, Mol. Pharmacol. 62 (2002) 864-872.

[7] J. Rejman, A. Bragonzi, M. Conese, Role of clathrin- and caveolae-mediated endocytosis in gene transfer mediated by lipo- and polyplexes, Mol. Ther. 12 (2005) 468-474, http://dx.doi.org/10.1016/j.ymthe.2005.03.038.

[8] R.K. Keswani, M. Lazebnik, D.W. Pack, Intracellular trafficking of hybrid gene delivery vectors, J. Control. Release 207 (2015) 120-130, http://dx.doi.org/10 1016/j.jconrel.2015.04.015.

[9] P.L. Felgner, T.R. Gadek, M. Holm, R. Roman, H.W. Chan, M. Wenz, J.P. Northrop, G.M. Ringold, M. Danielsen, Lipofection: a highly efficient, lipid-mediated DNA-transfection procedure, Proc. Natl. Acad. Sci. U. S. A. 84 (1987) 7413-7417.

[10] S.D. Conner, S.L. Schmid, Regulated portals of entry into the cell, Nature 422 (2003) 37-44, http://dx.doi.org/10.1038/nature01451.

[11] C. Lamaze, S.L. Schmid, The emergence of clathrin-independent pinocytic pathways, Curr. Opin. Cell Biol. 7 (1995) 573-580, http://dx.doi.org/10.1016/ 0955-0674(95)80015-8.

[12] K. Takei, V. Haucke, Clathrin-mediated endocytosis: membrane factors pul the trigger, Trends Cell Biol. 11 (2016) 385-391, http://dx.doi.org/10.1016/ S0962-8924(01)02082-7.

[13] F.R. Maxfield, T.E. McGraw, Endocytic recycling, Nat. Rev. Mol. Cell Biol. 5 (2004) 121-132, http://dx.doi.org/10.1038/nrm1315.

[14] S. Matveev, X. Li, W. Everson, E.J. Smart, The role of caveolae and caveolin in vesicle-dependent and vesicle-independent trafficking, Adv. Drug Deliv. Rev. 49 (2001) 237-250, http://dx.doi.org/10.1016/S0169-409X(01)00138-7.

[15] J. Harris, D. Werling, J.C. Hope, G. Taylor, C.J. Howard, Caveolae and caveolin in immune cells: distribution and functions, Trends Immunol. 23 (2016) 158-164, http://dx.doi.org/10.1016/S1471-4906(01)02161-5.

[16] I.A. Khalil, K. Kogure, H. Akita, H. Harashima, Uptake pathways and subsequent intracellular trafficking in nonviral gene delivery, Pharmacol. Rev. 58 (2006) 32-45, http://dx.doi.org/10.1124/pr.58.1.8.32.

[17] A. Ferrari, V. Pellegrini, C. Arcangeli, A. Fittipaldi, M. Giacca, F. Beltram, Caveolae-Mediated internalization of extracellular HIV-1 tat fusion proteins visualized in real time, Mol. Ther. 8 (2003) 284-294, http://dx.doi.org/10. 1016/S1525-0016(03)00122-9.
[18] J.A. Swanson, C. Watts, Macropinocytosis, Trends Cell Biol. 5 (1995) 424-428, http://dx.doi.org/10.1016/S0962-8924(00)89101-1.

[19] O. Meier, K. Boucke, S.V. Hammer, S. Keller, R.P. Stidwill, S. Hemmi, U.F. Greber, Adenovirus triggers macropinocytosis and endosomal leakage together with its clathrin-mediated uptake, J. Cell Biol. 158 (2002) 1119-1131, http://dx.doi.org/10.1083/jcb.200112067.

[20] K. Sandvig, S. Pust, T. Skotland, B. Van Deurs, Clathrin-independent endocytosis: mechanisms and function, Curr. Opin. Cell Biol. 23 (2011) 413-420, http://dx.doi.org/10.1016/j.ceb.2011.03.007.

[21] P. Alves, A.A. Hugo, E.E. Tymczyszyn, A.F. Ferreira, R. Fausto, P.F. Pérez, J.F.J. Coelho, P.N. Simões, A. Gómez-zavaglia, Effect of

cholesterol-poly( $N, N$-dimethylaminoethyl methacrylate) on the properties of stimuli-responsive polymer liposome complexes, Colloids Surf. B: Biointerfaces 104 (2013) 254-261.

[22] F. Szoka, D. Papahadjopoulos, Procedure for preparation of liposomes with large internal aqueous space and high capture by reverse-phase evaporation, Proc. Natl. Acad. Sci. U. S. A. 75 (1978) 4194-4198 http://www.ncbi.nlm.nih. gov/pmc/articles/PMC336078/.

[23] M. Werle, K. Hironaka, H. Takeuchi, H. Hoyer, Development and in vitro characterization of liposomes coated with thiolated poly(acrylic acid) for oral drug delivery, Drug Dev. Ind. Pharm. 35 (2009) 209-215, http://dx.doi.org/10. $1080 / 03639040802244326$.

[24] K. Joo, L. Xiao, S. Liu, Y. Liu, C.-L. Lee, P.S. Conti, M.K. Wong, Z. Li, P. Wang, Crosslinked multilamellar liposomes for controlled delivery of anticancer drugs, Biomaterials 34 (2013) 3098-3109.

[25] C. Lo, W. Liao, C. Wu, J. Lee, M. Sun, H. Yang, W. Tsai, Y. Chang, W. Chen, Synergistic effect of PEI and PDMAEMA on transgene expression in vitro, Langmuir 31 (2015) 6130-6136, http://dx.doi.org/10.1021/acs.langmuir. 5 b00520.

[26] J. Saraste, G.E. Palade, M.G. Farquhar, Temperature-sensitive steps in the transport of secretory proteins through the Golgi complex in exocrine pancreatic cells, Proc. Natl. Acad. Sci. U. S. A. 83 (1986) 6425-6429.

[27] S. Simoes, V. Slepushkin, P. Pires, R. Gaspar, M. Pedroso de Lima, N. Du, Mechanisms of gene transfer mediated by lipoplexes associated with targeting ligands or pH-sensitive peptides, Gene Ther. 6 (1999) 1798-1807.

[28] R. Bajoria, S.R. Sooranna, S.F. Contractor, Endocytotic uptake of small unilamellar liposomes by human trophoblast cells in culture, Hum. Reprod. 12 (1997) 1343-1348, http://dx.doi.org/10.1093/humrep/12.6.1343.

[29] R.A. Jones, M.H. Poniris, M.R. Wilson, pDMAEMA is internalised by endocytosis but does not physically disrupt endosomes, J. Control. Release 96 (2006) 379-391, http://dx.doi.org/10.1016/j.jconrel.2004.02.011.

[30] P.A. Orlandi, P.H. Fishman, Filipin-dependent inhibition of cholera toxin: evidence for toxin internalization and activation through caveolae-like domains, J. Cell Biol. 141 (1998) 905-915.

[31] S. Zhang, J. Li, G. Lykotrafitis, G. Bao, S. Suresh, Size-dependent endocytosis of nanoparticles, Adv. Mater. 21 (2009) 419-424, http://dx.doi.org/10.1002/ adma.200801393.

[32] G.J. Doherty, H.T. McMahon, Mechanisms of endocytosis, Annu. Rev. Biochem. 78 (2009) 857-902, http://dx.doi.org/10.1146/annurev.biochem.78.081307. 110540.

[33] M. Huang, Z. Ma, E. Khor, L.Y. Lim, Uptake of FITC-chitosan nanoparticles by A549 cells, Pharm. Res. 19 (2002) 1488-1494, http://dx.doi.org/10.1023/ A:1020404615898. 